\title{
Development and Hardware-in-the-Loop Testing of an Extended Kalman Filter for Attitude Estimation
}

\author{
Péter Bauer Computer and Automation Research Institute \\ Hungarian Academy of Sciences \\ Budapest, Hungary \\ Email: bauer.peter@sztaki.hu József Bokor Computer and Automation Research Institute \\ Hungarian Academy of Sciences \\ Budapest, Hungary
}

\begin{abstract}
This paper describes the development and hardware-in-the-loop testing of an Extended Kalman Filter (EKF) for attitude estimation. After literature review, a multimode solution to the estimation problem is introduced. It uses the sensor measurements optimally relying on the magnetic and acceleration data on the ground, and magnetic and GPS data in the air. An emergency aerial mode dealing with lost GPS data is also developed. The quaternion dynamic equations are chosen to represent system dynamics. Their special structure makes it possible to perform continuous-discrete transformation with a closed form solution of the Heun scheme. This can improve the prediction performance of the EKF. The observability of the system was examined using gridding of the state space in every modes of the filter. The computing steps of the new filter are summarized before presenting issues of implementation and testing. This article presents hardware-in-the-loop test results with simulated GPS losses. Comparison to another filter is also presented. The estimator was tested in real flights and performed as expected.
\end{abstract}

\section{INTRODUCTION}

In the recent years, the research on unmanned aerial vehicle (UAV) guidance and navigation algorithms gets more and more attention. In 2006, a research cooperation started between University of Minnesota (UofM), University of Sannio (UofS) and Computer and Automation Research Institute (CARI). The goal of this cooperation was to develop and build an aircraft platform on which different guidance and control algorithms can be implemented and tested in an easy and cost effective manner. The project uses the E-flite Ultrastick 25e which is a small radio controlled ( $\mathrm{RC}$ ) airplane (see [1] for more details).

In this project, the first step was to develop simple PID controllers for stabilization and path tracking flight. The application of stabilization and tracking controllers requires known aircraft attitude during flight. This can not be measured, only estimated from other measured data.

In the project, the Extended Kalman Filter (EKF) - proposed in [2] - was used to estimate attitude Euler angles. The hardware-in-the-loop (HIL) tests showed that the performance of this filter is not satisfactory. In the HIL test, the aircraft and sensor unit were replaced by Matlab Simulink models, but the same hardware (i.e., microcontroller and RC system) as installed onboard was used. An advantage of this testing is that the estimated Euler angles can be compared with the ones calculated in Matlab.
The poor performance of the filter motivated the authors to search for better solutions which can perform well. The goal was to develop an attitude estimator which can be used from aircraft preparation (before take off) to aircraft shutdown (after landing).

The outline of the paper is as follows: Section II overviews the basic decisions (based on literature review) done at the beginning of development and shows the structure of the developed algorithm. Section III briefly introduces the theoretical steps concerning the equations of the filter. Section IV outlines testing and tuning of the algorithm and presents the HIL simulation results. Finally, the conclusion summarizes the results.

\section{Decisions ABout THE APPLIED METHODS AND STRUCTURE OF THE ATTITUDE ESTIMATOR}

The first task in the development of a state estimator is to select the sensors to be used or select the (required) measured quantities if the sensor unit is given. In this project, the Crossbow $\mu N A V$ low cost MEMS sensor (see [3]) is used.

This unit measures acceleration (accel.), magnetic (magn.) and angular rate vector in the aircraft body coordinate system (coord. sys.) and the barometric and Pitot tube pressures. Furthermore, it can receive GPS signals. From the received GPS signals the aircraft velocity and position can be obtained (in earth coord. sys.). All measured quantities were calibrated and tested. GPS signal is broadcast with $4 \mathrm{~Hz}$ every other data with $50 \mathrm{~Hz}$. Based on this unit, loosely or tightly coupled GPS/INS solutions, or a standalone INS solution can be built.

In the original estimator (see [2]) - and in many estimators ([4], [5], [6]) - the accel. and magn. vectors were used as measured outputs. However, the accel. vector can only be used to estimate aircraft attitude if it coincides with the direction of Earth's gravity vector. This is only true if aircraft stan ds on the ground, or makes no maneuvers, otherwise the dynamical accelerations highly distort the measurement. Despite this distorting effect, the aforementioned assumption is used in [2], [4], [5], [6], and only [4] and [5] mention that this can be used only in hovering or quasi hovering situations characterizing helicopters and quadrotors. The violation of this assumption could lead to the original observer's poor performance.

[6] proposes the correction of measured accel. with the dynamical part to get measurements closer to gravity vector. 
But this correction can not be completely achieved because body velocity components and their accelerations are usually unknown and so, only the indicated airspeed (IAS) measurement (from Pitot pressure) can be used. This correction was implemented and tested in conjunction with the original estimator, but did not cause significant performance improvement.

The best solution would be not to use the accel. measurements during flight of the aircraft. However, the estimation of the rotation matrix requires two independent vector measurements. It was pointed out in [7] that the system becomes again observable if one considers the magn. and GPS measurements together. So, by relying on these measurements an acceptable solution can be achieved.

However, the GPS velocity and position measurements are useless in low speed regions of the flight (before take off and after landing), while the magn. measurements are equally useful on ground and in air. In summary, one can well use magn. and accel. measurements on ground and magn. and GPS measurements in air. This leads to the idea of developing a multi-mode filter, that can switch between these signal pairs. This concept was used in [8] to satisfy fault tolerant needs. Here, it is used to improve estimation performance.

Having decided about the required sensor measurements, one has to think about the measurement errors. Acceleration, magnetic and angular rate measurements are usually corrupted by bias drift and measurement noise, while GPS measurement is only corrupted by noise. The noises are usually assumed to be zero-mean Gaussian. Such noises can be filtered out by a well-tuned estimator. The biases are usually slowly varying, almost constant quantities. They can be estimated as states of the system if the augmented system is observable. It is proved in [5] that from accel. and magn. measurements only the rate gyro bias is observable. Thus, the multi-mode filter will only be able to estimate this bias.

As the next design step the suitable representation of the rotation matrix should be determined. For example, [4] and [6] estimates elements of the rotation matrix, [8] uses Euler angles while [2], [5], [7] use quaternion representation. In the considered case, the quaternion representation was selected, because it never becomes singular and its dynamical equation is very simple which leads to a more accurate formulation of the filter (see Section III). However, the final outputs of the estimator are the three Euler angles.

The next decision to make is the selection of the nonlinear filtering method (estimator algorithm). [4] and [5] use nonlinear observers based on Lyapunov technique or Lie group theory, while [2], [6], [7] and [8] use EKF or its modifications. The EKF algorithm is selected, because the original estimator was an EKF (parts of the original code can be reused) and this algorithm is widely used and computationally viable.

In the original code, the EKF measurement update was done only at every second estimation step. This strategy has decreased the computational power needs so, it has been applied on the new EKF as well.

The next design step concerns the different modes of the filter and the switching strategy between them. The following modes of operation were selected:

1) MODE 1: Initialization of Euler angles based on magn. and accel. measurements (when aircraft stands undisturbed on the ground). This is required because the EKF is very sensitive to the initial conditions. The calculation of Earth's magnetic vector components (in earth coord. sys.) is also done. These are constant and required also in the subsequent modes (see (10)). The rate gyro bias values are also initialized. The initial quaternion is calculated from the Euler angles.

2) MODE 2: Ground mode before take off. Continuous update of estimates based on magn. and accel. measurements.

3) MODE 3: Aerial mode with GPS. Continuous update of estimates based on magn. and GPS measurements during flight.

4) MODE 4: Aerial mode without GPS. Continuous update of estimates based on magn. and IAS corrected accel. measurements during flight.

5) MODE 5: Ground mode after landing. Continuous update of estimates based on magn. and accel. measurements.

The described estimator modes of operation represent a loosely coupled INS/GPS solution. The switching conditions between the different modes are as follows:

1) MODE 1 to MODE 2: MODE 2 starts automatically after initialization which lasts for 10 seconds.

2) MODE 2 to MODE 3: The switching happens when $a_{x}<-0.32 g, \delta_{t h}>80 \%$ and the estimator is in MODE 2. Here the goal is to establish the time of take off. This is characterized by large longitudinal acceleration (in absolute value) and full throttle position. The accel. and throttle limits were determined from concrete flight data. The limits were selected to avoid false switching. The third condition is required to avoid in-flight switching when aircraft does maneuvers with large $a_{x}$ accelerations.

3) MODE 3 to MODE 4: This is required if there is no GPS signal or erroneous signal arrives for at least 3 seconds. This time limit is required to avoid frequent switching. The time value was selected examining the divergence properties of the filter with purely magn. measurements to make it possible to quickly converge to the real angles in MODE 4.

4) MODE 4 to MODE 3: If valid GPS data arrives again.

5) MODE 3 to MODE 5: For this change, the absolute GPS velocity and flight time are considered. If the velocity is below $0.2 \mathrm{~m} / \mathrm{s}$ and flight time is above $120 \mathrm{~s}$ the system switches. The flight time limit is required because otherwise the system switches into MODE 5 during take off. The velocity limit was selected from several manual flight tests.

Unfortunately, it is not possible to switch from MODE 4 to MODE 5 because GPS is not available in MODE 4. Experiences show that it would be better to use the static pressure for switching as it can be applied also in MODE 4.

The flowchart of the EKF program with the different modes of operation can be seen in Figure 1. odd is a logical variable 


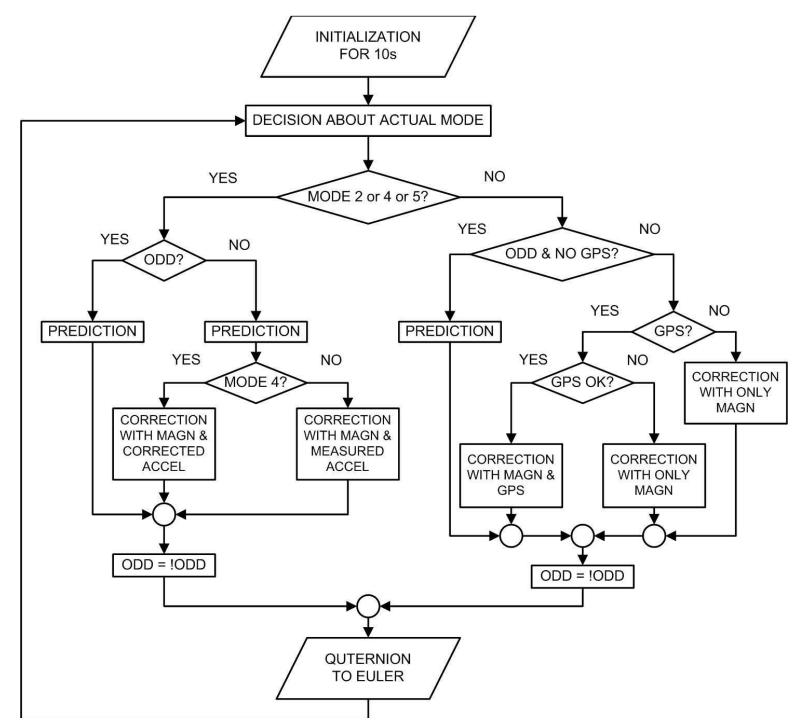

Fig. 1. EKF program flowchart

that can be 1 or 0 and it shows whether the actual calculation step is odd or even. If valid GPS data arrives in MODE 3 the correction step is done irrespective of the value of odd.

\section{THEORETICAL DEVELOPMENT OF THE FILTER EQUATIONS}

Firstly, the initialization formulae are presented. The mean measured values of acceleration, magnetic and angular rate data are used. These means should be calculated using a recursive formula to avoid the unnecessary storage of input values:

$$
\bar{v}_{1}=v_{1}, \quad \bar{v}_{k+1}=\bar{v}_{k} \frac{k}{k+1}+v_{k+1} \frac{1}{k+1}
$$

Here, $v$ represents the vector of measured data and $\bar{v}$ is the computed mean.

The initial roll and pitch angles can be calculated from the mean acceleration values as follows:

$$
\phi=\arctan \left(\frac{\bar{a}_{y}}{\bar{a}_{z}}\right), \quad \theta=\arcsin \left(-\bar{a}_{x}\right)
$$

Using these angles the body magnetic vector $\left(V^{B}\right)$ can be transformed into earth coord. sys. $\left(V^{E^{\prime}}\right)$ assuming zero yaw angle. This way, the magnetic yaw angle $\left(\psi^{\prime}\right)$ can be calculated from the horizontal magnetic components in earth coord. sys. Finally, the yaw angle corrected with magnetic declination will be the real yaw angle $(\psi)$ of the aircraft:

$$
\begin{aligned}
& V^{E^{\prime}}=\left.T_{\psi}^{T} T_{\theta}^{T} T_{\phi}^{T}\right|_{\psi=0} V^{B} \\
& \psi^{\prime}=\arctan 2\left(\frac{-V^{E^{\prime}}(2)}{V^{E^{\prime}}(1)}\right), \quad \psi=\psi^{\prime}+D \\
& V^{E}=T_{\psi}^{T} T_{\theta}^{T} T_{\phi}^{T} V^{B}
\end{aligned}
$$

In (3), the Earth's magnetic vector $\left(V^{E}\right)$ is calculated in earth coord. sys. $D$ is the magnetic declination.
After deriving these formulae tests were carried out considering collected data before take off and after landing. These tests showed that the yaw rate bias can be considered constant during the whole flight, while the other biases vary. This is a really advantageous situation, as the original EKF had a tendency to estimate the whole yaw rate as bias, particularly, if the aircraft flew circles. This problem can be avoided considering only the roll and pitch rate biases in the filter.

The initial quaternion can be calculated from the initial Euler angles as given in [9].

Second, the dynamic and measurement equations of the EKF are derived. The continuous time (CT) differential equation of the quaternion vector in a rotating coordinate frame is as follows:

$$
\dot{q}=-\frac{1}{2}\left[\begin{array}{cccc}
0 & \bar{P} & \bar{Q} & \bar{R} \\
-\bar{P} & 0 & -\bar{R} & \bar{Q} \\
-\bar{Q} & \bar{R} & 0 & -\bar{P} \\
-\bar{R} & -\bar{Q} & \bar{P} & 0
\end{array}\right] q
$$

In (4), $\bar{P}$ represents the real roll rate which means the measured roll rate corrected with the bias and zero mean Gaussian white noise, that is, $\bar{P}=P-b_{P}-v_{P}$. The same convention is used for the pitch and yaw rate variables.

Assuming that the yaw rate bias is constant, and reorganizing (4) results in the following CT dynamic equations with parameter and state dependent coefficient matrices. The system states are the quaternion and the time varying bias values:

$$
\begin{aligned}
\dot{q} & =A_{1}(\rho) q+A_{2}(q) b+V_{1}(q) v^{q}= \\
= & A(\rho, b) q+V_{1}(q) v^{q} \\
\text { where } & \rho=\left[\begin{array}{lll}
P & Q & R-b_{R}
\end{array}\right]^{T} \quad b=\left[\begin{array}{ll}
b_{P} & b_{Q}
\end{array}\right]^{T} \\
& v^{q}=\left[\begin{array}{lll}
v_{P} & v_{Q} & v_{R}
\end{array}\right]^{T}
\end{aligned}
$$

The dynamics of the slowly varying biases can be modelled as a system driven by zero-mean Gaussian white noise:

$$
\dot{b}=v^{b} \quad \text { where } v^{b}=\left[\begin{array}{ll}
v_{b P} & v_{b Q}
\end{array}\right]^{T}
$$

Equations (5) and (6) are the CT equations of the system dynamics. They are state and parameter dependent and consequently nonlinear. From these system of equations a discrete time (DT) linearized system should be derived to make the Kalman filter algorithm applicable. The discretization can be done using some integration formula. The simplest one is the Euler (rectangular) the more accurate is the Heun (trapezoidal) scheme. Originally, the Heun formula needs the prediction of future state values (see [10]), but for the quaternion dynamics a closed form and therefore more accurate solution exists thanks to the special structure of equations.

$$
\begin{aligned}
& q_{k+1} \approx q_{k}+\frac{\dot{q}_{k}+\dot{q}_{k+1}}{2} d t= \\
& =q_{k}+\frac{d t}{2}\left(A_{1}\left(\rho_{k}\right) q_{k}+A_{2}\left(q_{k}\right) b_{k}+V_{1}\left(q_{k}\right) v_{k}^{q}\right)+ \\
& +\frac{d t}{2}\left(A\left(\rho_{k+1}, b_{k+1}\right) q_{k+1}+V_{1}\left(q_{k+1}\right) v_{k+1}^{q}\right) \\
& b_{k+1} \approx b_{k}+\frac{v_{k}^{b}+v_{k+1}^{b}}{2} d t
\end{aligned}
$$


Here, $d t$ is the discrete time step and indexes $k$ and $k+1$ denote the sampled data values. Assuming $b_{k+1}=b_{k}$ (slowly varying bias value), considering the predicted quaternion $\bar{q}_{k+1}$ with some approximation in the $V_{1}$ terms, introducing fictitious noises $\left(\bar{v}_{k+1}^{q}, \quad \bar{v}_{k+1}^{b}\right)$ and reordering the terms in (7) results in:

$$
\begin{aligned}
& \underbrace{\left(I-\frac{d t}{2} A\left(\rho_{k+1}, b_{k}\right)\right)}_{M_{k+1}^{+}} q_{k+1}=\underbrace{\left(I+\frac{d t}{2} A_{1}\left(\rho_{k}\right)\right)}_{M_{k}^{-}} q_{k}+ \\
& +\frac{d t}{2} A_{2}\left(q_{k}\right) b_{k}+d t V_{1}\left(\bar{q}_{k+1}\right) \bar{v}_{k+1}^{q} \\
& b_{k+1}=b_{k}+d t \bar{v}_{k+1}^{b}
\end{aligned}
$$

The EKF can be programmed in a way which ensures that the measured $\rho_{k+1}$ parameter vector is available at the time of state prediction. This means that (8) has a closed form solution, if $M_{k+1}^{+}$is invertible. It can be proven that it is always invertible and its inverse can be calculated in closed form. This way, the DT state dynamic equation of the filter is as follows:

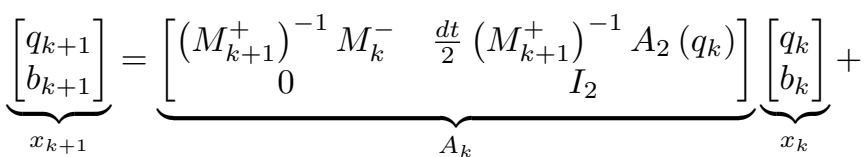

$$
\begin{aligned}
& +\underbrace{\left[\begin{array}{cc}
d t\left(M_{k+1}^{+}\right)^{-1} V_{1}\left(\bar{q}_{k+1}\right) & 0 \\
0 & d t I_{2}
\end{array}\right]}_{V_{k}}\left[\begin{array}{c}
\bar{v}_{k+1}^{q} \\
\bar{v}_{k+1}^{b}
\end{array}\right]
\end{aligned}
$$

After deriving the DT state equations, the output equations should be derived. The first equation (10) is the measurement of magn. vector in body coord. sys. The nonlinear equation and its Jacobian are as follows:

$$
V^{B}=T^{E B}(q) V^{E}=h_{1}\left(q, V^{E}\right), \quad C_{1}=\frac{\partial h_{1}\left(q, V^{E}\right)}{\partial q}
$$

Here, $V^{E}$ (Earth's magnetic vector) is known from the initialization and can be assumed to be constant during the 1015 minutes flight duration. $T^{E B}(q)$ represents the quaternion based rotation matrix from earth to body coord. sys.

The second equation (11) is the measurement of Earth's gravity vector in body coord. sys:

$$
a^{B}=T^{E B}(q)\left[\begin{array}{lll}
0 & 0 & 1
\end{array}\right]^{T}=h_{2}(q), \quad C_{2}=\frac{d h_{2}(q)}{d q}
$$

Here, $a^{B}$ is approximately corrected with the dynamic acceleration in MODE 4, otherwise it is used as measured.

The last output is the azimuth angle from GPS (12). This can be calculated either from the GPS position or velocity. GPS velocity measurements are usually more accurate and the azimuth angle can be calculated from their instantaneous values. Therefore, the velocity components are used. The nonlinear output equation (with Evel and $\mathrm{Nvel}$ as east and north GPS velocities) and the Jacobian are:

$$
\begin{aligned}
& \psi=\arctan 2\left(\frac{\text { Evel }}{\text { Nvel }}\right)= \\
& =\arctan \left(\frac{T^{E B}(q)(1,2)}{T^{E B}(q)(1,1)}\right)=h_{3}(q), \quad C_{3}=\frac{d h_{3}(q)}{d q}
\end{aligned}
$$

Here, $T^{E B}(q)(1, i)$ are selected elements from the rotation matrix.

This way the output matrix of the DT linearized equation in the different operation modes is as follows:

- $\operatorname{MODE} 2,4,5: C=\left[\begin{array}{ll}C_{1} & 0 \\ C_{2} & 0\end{array}\right]$

- $\operatorname{MODE} 3: C=\left[\begin{array}{ll}C_{1} & 0 \\ C_{3} & 0\end{array}\right] \quad$ or $C=\left[\begin{array}{ll}C_{1} & 0\end{array}\right]$

Here, the additional zero matrix blocks are required because the biases are also parts of the EKF state vector (see (9)). The structure of the output equation is shown below (with $w$ zeromean Gaussian measurement noise vector):

$$
y_{k+1}=C_{k+1} x_{k+1}+w_{k+1}
$$

After deriving the DT linearized equations, the observability of the system must be checked. This can be done by checking the observability of the linearized system in several points of the state space. The question is whether the system is observable in all the operation modes, or not. A set of Euler angles was used to grid the quaternion space:

$$
\begin{aligned}
& \phi=[-90: 10: 90 \quad-45 \quad 45] \quad[\mathrm{deg}] \\
& \theta=\left[\begin{array}{lll}
-60: 10: 60 & -45 & 45
\end{array}\right] \quad[\mathrm{deg}] \\
& \psi=[-180: 10: 180 \quad-45 \quad 45] \quad[\mathrm{deg}]
\end{aligned}
$$

These values were selected to cover the flight envelope of a non-aerobatic UAV. Observability check with zero angular rates and biases and also with nonzero ones was performed. In the first case, the system was not observable if only magnetic measurements were used. In the second case, it was always observable. But it is known that the orientation can not be determined from only one vector measurement. As a consequence, there can be only local observability in case of pure magnetic measurements.

Finally, the order of calculations should be decided upon. The structure of EKF algorithm can be found e.g. in [2]. The computing modes and steps of the current algorithm are as follows:

1) STEP $k$ ONLY PREDICTION

- $\left(M_{k}^{+}\right)^{-1} \rightarrow A_{k-1}$

- $\bar{x}_{k}=A_{k-1} \hat{x}_{k-1} \rightarrow V_{1}\left(\bar{q}_{k}\right) \Rightarrow V_{k-1}$

- $\bar{X}_{k}=A_{k-1} X_{k-1} A_{k-1}^{T}+V_{k-1} V V_{k-1}^{T}$

- $\hat{x}_{k}=\bar{x}_{k}, X_{k}=\bar{X}_{k}$

- $M_{k}^{-}, \quad \frac{d t}{2} A_{2}\left(\hat{q}_{k}\right)$

2) STEP $k+1$ PREDICTION WITH CORRECTION

- $\left(M_{k+1}^{+}\right)^{-1} \rightarrow A_{k}$

- $\bar{x}_{k+1}=A_{k} \hat{x}_{k} \rightarrow V_{1}\left(\bar{q}_{k+1}\right) \Rightarrow V_{k}$

- $\bar{X}_{k+1}=A_{k} X_{k} A_{k}^{T}+V_{k} V V_{k}^{T}$

- $C_{k+1}\left(\bar{q}_{k+1}\right)$

- $K_{k+1}=\bar{X}_{k+1} C_{k+1}^{T}\left[C_{k+1} \bar{X}_{k+1} C_{k+1}^{T}+W\right]^{-1}$ 
- $\hat{x}_{k+1}=\bar{x}_{k+1}+K_{k+1}\left[y_{k+1}-h\left(\bar{x}_{k+1}\right)\right]$

- $X_{k+1}=\left[I-K_{k+1} C_{k+1}\right] \bar{X}_{k+1}$

- $M_{k+1}^{-}, \quad \frac{d t}{2} A_{2}\left(\hat{q}_{k+1}\right)$

Here, $V$ is the angular rate and gyro bias noise covariance matrix, while $W$ is the noise covariance matrix of output measurements. Note that its dimension is different in the different operation modes. $X$ is the state estimation error covariance matrix, (.) denotes predicted, while $(\hat{.})$ denotes corrected values.

\section{TUNING AND TESTING OF THE FILTER}

Tuning and testing alternated during the filter development. The final filter weights (noise covariance values) were selected after several iterations, and it turned out that MODE 4 requires magnetic and acceleration noise covariances different from those in MODE 2 and 5. This is not surprising if we consider the different function of these operation modes.

The whole process was performed as follows:

1) Step by step implementation and testing of the EKF code in Matlab and tuning of parameters using real flight test data.

2) Code optimization considering the sparse and special structure of the system matrices (see (9))

3) $C$-mex file implementation of the code and comparison with $m$ file results in Matlab for the same data. The $C$ code should give exactly the same results as the $m$ and this was achieved.

4) Implementation onboard the MPC555 microcontroller. Indoor and outdoor tests and fine tuning of weights in ground mode.

5) HIL tests and fine tuning of weights in aerial mode.

6) Several successful flight tests were conducted with autopilots using the new EKF (NEKF), but evaluation of flight test data is out of the scope of this paper.

Now, the HIL test results with the original (UofM) and new (NEKF) estimators are compared. This is the only possibility to evaluate the performance of the NEKF as the real Euler angles are not measured in flight. The estimated Euler angles in mixed manual and autopilot flight tests (with several simulated GPS losses) are shown in Figs. 2-4. The steps of the HIL test with NEKF were as follows (note that the UofM filter does not use GPS):

- Start with manual flight, lose GPS after some time.

- GPS re-appears, then start roll doublet tracking, lose GPS during tracking.

- Switch back GPS during manual flight.

- Switch auto mode again, lose GPS during stabilization phase.

- Switch back GPS during stable (autopilot) flight.

- Lose and switch back GPS during roll doublet tracking maneuver.
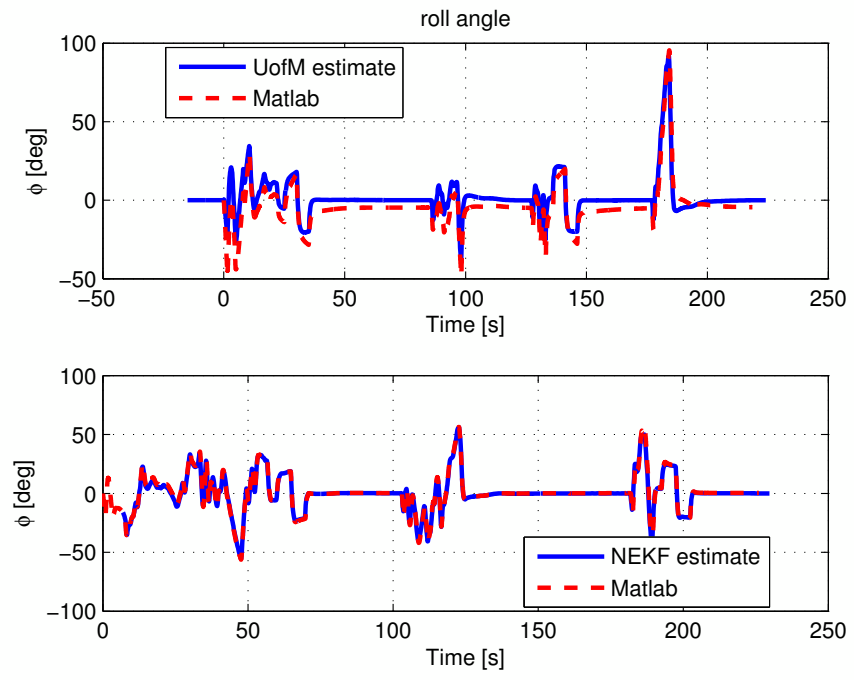

Fig. 2. Estimation of roll angles
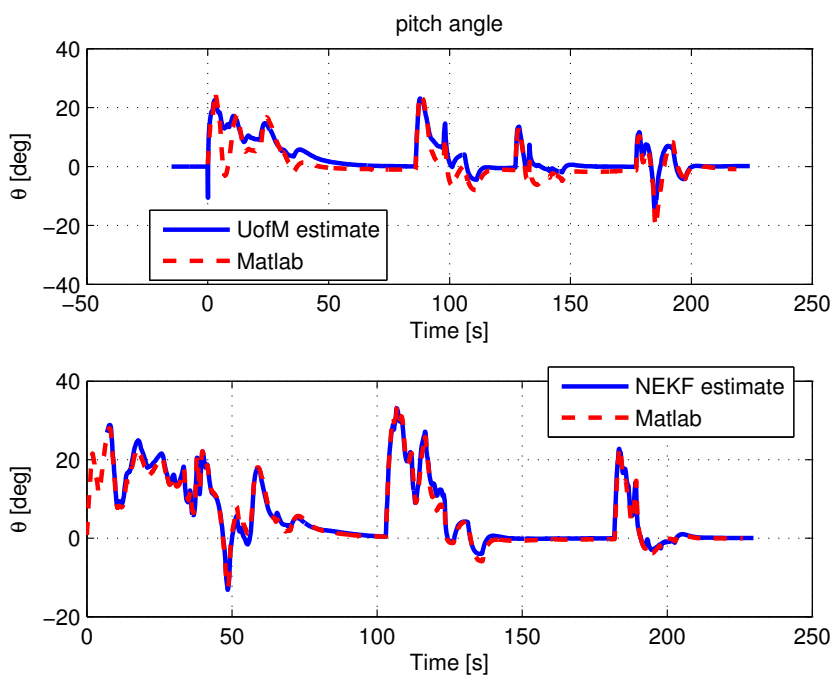

Fig. 3. Estimation of pitch angles

The dashed lines represent the 'real' Euler angle values computed by Matlab while the continuous lines represent the estimated ones. It is obvious from all figures that the new EKF has better performance than the original one, despite the simulated GPS losses (for about 20 / 30 / 40 seconds).

Fig. 5 shows the absolute values of the estimation errors for the original (UofM) and new (NEKF) codes. In case of the new code, these errors are much smaller.

In essence, the goal of the development was clearly achieved, a new EKF was successfully developed which outperforms the original one. This was verified on real flight data and during flight tests.

\section{CONCLUSION}

This paper presented the development and HIL testing of an attitude EKF. The work was motivated by unsatisfactory experiences from a previous EKF solution. After literature review, a multi-mode solution was proposed which uses the sensor 

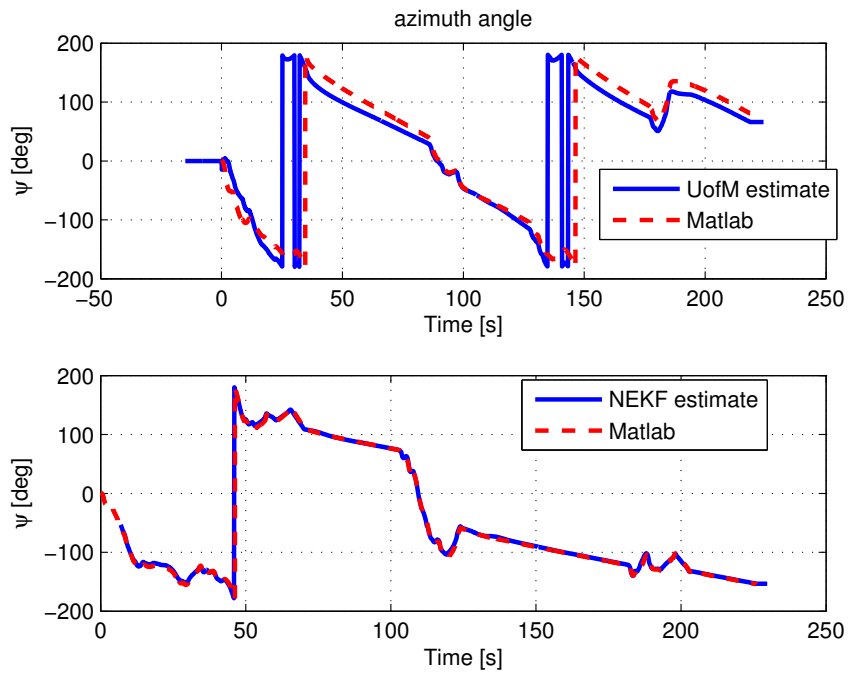

Fig. 4. Estimation of azimuth angles

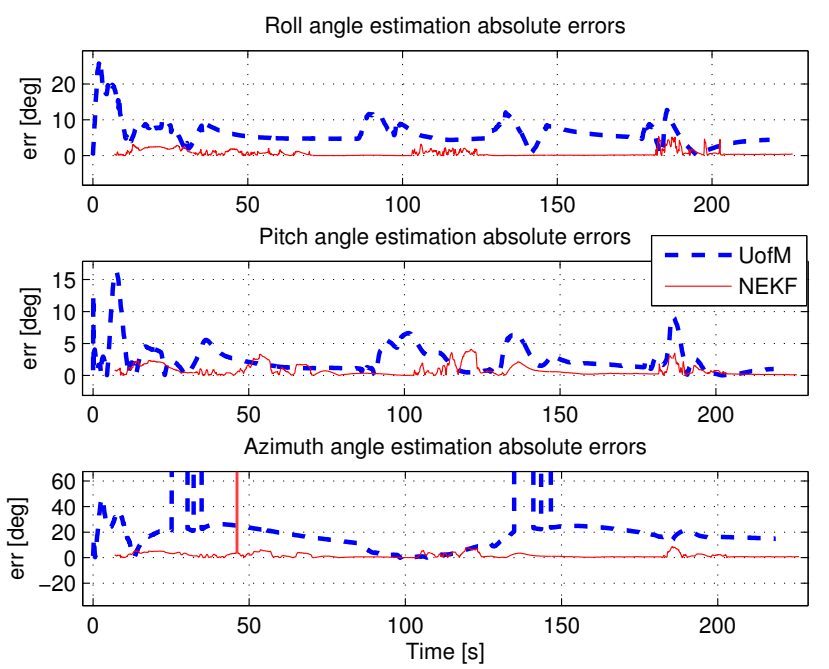

Fig. 5. Absolute estimation errors

measurements optimally. This means the use of magnetic and acceleration data in ground modes and the use of magnetic and GPS data in aerial mode. An emergency aerial mode for the cases with lost GPS is also developed.

It was pointed out that the yaw rate bias can be considered constant and therefore, only the other two (roll and pitch) biases should be estimated.

The well-known and widely used EKF algorithm was selected to solve the estimation problem. A quaternion representation of the rotation matrix was applied. Besides nonsingularity, this made it possible to derive a closed-form solution for the Heun scheme used in the CT-DT transformation of the system equations.

The used equations and algorithm steps were presented, together with a flowchart of the whole estimator program.

After checking the observability of the system in the different modes using a state space gridding technique (considering the flight envelope of a non aerobatic small UAV), the steps of the tuning and testing procedure were briefly described.

Finally, the evaluation of estimator performance was done through HIL simulations by comparing it to another algorithm and testing with simulated GPS losses.

Real flight tests of the algorithm were also performed but these will form a topic of another article with the more detailed evaluation of results achieved.

\section{ACKNOWLEDGMENT}

The authors gratefully acknowledge to the support by the Hungarian National Scientific Research Fund (OTKA CNK 78168) and by the Control Engineering Research Group of HAS at Budapest University of Technology and Economics.

\section{REFERENCES}

[1] P. Y. Chai, UAV Research group. University of Minnesota http://www.aem.umn.edu/ uav/index.html. (Cited 31 August 2010), 2006.

[2] J. S. Jang and D. Liccardo, Automation of small UAVs using low cost MEMS sensor and embedded computing platform, in Proc. of the 25th Digital Avionics Systems Conference, 15. October 2006.

[3] MNAV 100CA User's Manual, Crossbow Technology Inc., San Jose, USA, 2005.

[4] N. Metni, J.-M. Pfimlin, T. Hamel and P. Soures, Attitude and gyro bias estimation for a VTOL UAV, Control Engineering Practice, Vol. 14, pp. 1511-1520, March 2006.

[5] P. Martin and E. Salan, Design and implementation of a low-cost observer-based attitude and heading reference system, Control Engineering Practice, Vol. 18, pp. 712-722, July 2010.

[6] C. Liu, Z. Zhou and X. Fu, Attitude determination for MAVs using a Kalman filter, Tsinghua Science and Technology, Vol. 13, Nr. 5, pp. 593-597, October 2008.

[7] J. Bijker and W. Steyn, Kalman filter configurations for low-cost loosely integrated inertial navigation system on an airship, Control Engineering Practice, Vol. 16, pp. 1509-1518, June 2008.

[8] D. J. Allerton and H. Jia, Redundant Multi-Mode Filter for a Navigation System, IEEE Transactions on Aerospace and Electronic Systems, Vol. 43, No. 1, pp. 371-390, January 2007.

[9] Wikipedia, Conversion between quaternions and Euler angles http://en.wikipedia.org/wiki/Conversion_ between_ quaternions_ and_ Euler_angles. (Cited 22. September 2010), 2005.

[10] T. Mazzoni, Computational aspets of continuous-discrete extended Kalman-filtering, Fern Universitat, Hagen, Germany, 23. April 2007. 